\title{
GASTO PÚBLICO, INVERSIÓN Y CRECIMIENTO EN MÉXICO, 1980-2014
}

\author{
Josefa Carolina Fortuno Hernández* \\ Heri Óscar Landa Díaz
}

(Recibido: abril 2014/Aceptado: agosto 2014)

\section{Resumen}

Desde los años ochenta, México ha administrado sus finanzas públicas con base en el enfoque de disciplina fiscal sugerido por la teoría ortodoxa, cuyo objetivo primordial es el equilibrio en las finanzas públicas. Por consiguiente, la política fiscal ha sido restrictiva y su efecto ha disminuido la demanda agregada, en general, y la inversión pública, en particular. Como consecuencia, la economía ha mostrado un lento crecimiento y una tendencia al estancamiento en el largo plazo. El objetivo de este artículo es analizar el impacto de esta política fiscal restrictiva en el crecimiento económico durante el periodo 1980-2014. En esta etapa la economía mexicana ha experimentado recurrentes crisis, ajustes recesivos de estabilización macroeconómica con austeridad fiscal, liberalización económica y finanzas públicas "sanas" (control del déficit fiscal primario). Con el propósito de contrastar los resultados macroeconómicos de esta política de finanzas públicas sanas con los resultados del periodo conocido como desarrollo estabilizador (1959-1970), hemos incluido datos de etapas de desarrollo anteriores a la instauración de las finanzas públicas sanas.

Clasificación JEL: E61, E62, E22.

Palabras clave: finanzas públicas, gasto, inversión.

* Profesora de la Facultad de Economía de la Universidad Veracruzana <jfortuno@uv.mx>y Pos-doctorante en la División de Estudios de Posgrado de la Facultad de Economía de la UNAM.

* Pos-doctorante en la División de Estudios de Posgrado de la Facultad de Economía de la UNAM, $<$ hold77@hotmail.com>. 


\section{Abstract}

México has managed its public finance based on the fiscal discipline approach since the 1980s. This approach's main objective is the balance of public finance. Consequently, fiscal policy has been restrictive and its effect has decreased aggregate demand, in general, and public investment in particular. As a result, the economy has shown slow growth and a tendency to stagnation in the long term. The purpose of this paper is to analyze the impact of restrictive fiscal policy on economic growth during 1980-2008. The Mexican economy has experienced recurring crises, recessionary adjustments of macroeconomic stabilization with fiscal austerity, economic liberalization and healthy public finance (control of the primary fiscal deficit). In order to compare the macroeconomic performance of this policy of sound public finance with the results of the period known as stabilizing development (1959-1970), we include data from the latter period.

JEL Classification: E61, E62, E22.

Keywords: public Finance, public expenditure, investment.

\section{Introducción}

México ha administrado sus finanzas públicas con base en el enfoque de disciplina fiscal sugerido por la teoría ortodoxa desde los años ochenta. El objetivo primordial de este enfoque es el equilibrio en las finanzas públicas. Por consiguiente, la política fiscal ha sido restrictiva y su efecto ha disminuido la demanda agregada, en general, y la inversión pública, en particular. Como consecuencia, la economía ha mostrado un lento crecimiento y una tendencia al estancamiento en el largo plazo.

En este artículo nos proponemos analizar cuál fue el impacto de la política fiscal restrictiva en el crecimiento económico durante el periodo 1980-2014. En esta etapa la economía mexicana ha experimentado recurrentes crisis, ajustes recesivos de estabilización macroeconómica con austeridad fiscal, liberalización económica y finanzas públicas sanas (control del déficit fiscal primario). Con el propósito de contrastar los resultados macroeconómicos de esta política de finanzas públicas sanas con los resultados del periodo conocido como desarrollo estabilizador (1959-1970), hemos incluido datos de etapas de desarrollo anteriores a la instauración de la política de finanzas "sanas". 
Además, hacemos hincapié en que las finanzas públicas en México podrían desempeñar un papel relevante en el financiamiento del desarrollo si se adoptara un enfoque distinto al prevaleciente. Por ejemplo, si se permitiera que la política fiscal contribuyera a estimular el mercado de trabajo, la productividad y el crecimiento económico y, de este modo, a superar -lo que hemos denominado- el estancamiento estabilizador que caracteriza a la economía mexicana desde hace más de veinte años.

El contexto histórico, político y social del periodo que estudiamos (19802014) corresponde al de la adopción, ascenso y predominio del modelo de liberalización comercial y financiera en la economía mexicana. En este contexto, el marco económico de las finanzas públicas es el de libre mercado, el de una política económica que estriba en la libre acción de la ley de la oferta y la demanda para conseguir los resultados económicos óptimos. Por lo tanto, el Estado no debe interferir en la economía mediante políticas fiscales activas y las finanzas públicas deben ceñirse al enfoque técnico del equilibrio presupuestal.

Nuestra hipótesis es la siguiente: la política de finanzas públicas sanas ha tenido un impacto negativo en la demanda agregada, en particular en la inversión pública. Dado que la tasa de crecimiento natural de la economía es endógena a la demanda, la política de "disciplina" fiscal ha tenido un impacto negativo en el crecimiento económico, configurando una economía de estancamiento estabilizador. Por lo tanto, una política fiscal que regule el gasto del gobierno puede contribuir a acelerar el ritmo de la actividad económica real. Por esta razón, nuestro objetivo principal es estimar la endogeneidad de la tasa de crecimiento natural de la economía mexicana con respecto a la demanda.

\section{Comportamiento macroeconómico de la economía mexicana: hechos estilizados}

Durante la fase del modelo de industrialización sustitutiva (IS) la economía mexicana registró un robusto crecimiento de la actividad económica, cimentado en la protección del mercado interno ${ }^{1}$ y la expansión de la demanda agregada. A pesar del buen desempeño económico observado durante este

\footnotetext{
${ }^{1}$ El Estado instrumentó aumentos progresivos del gasto público dirigido a las actividades industriales, así como una amplia batería de incentivos a la inversión y al ahorro, tales como: subsidios, exención de impuestos, reducción de los precios de bienes y servicios públicos o programas de depreciación acelerada de activos fijos.
} 
periodo (1960-2013), los resultados netos fueron negativos en términos de: elevados desequilibrios de las finanzas públicas, déficit sistemático en balanza comercial, incremento de la deuda externa, presiones inflacionarias y sobre el tipo de cambio.

Estas distorsiones dieron paso a la crisis económica y de balanza de pagos en 1976. Y en 1982 con el agotamiento del auge petrolero, tras la caída internacional de los precios del petróleo, la economía mexicana entró en un nuevo episodio de contracción económica que vulneró aún más las finanzas públicas, los niveles inflacionarios y la confianza para invertir en México.

En este contexto, en 1983 se inicia la transición hacia una estrategia de desarrollo dirigida por la demanda externa. Este nuevo proceso de desarrollo requeriría instrumentar políticas en dos vertientes: la estabilidad macroeconómica y el impulso del sector manufacturero de exportación. El primer eje se encaminaría al control de los desequilibrios económicos y financieros producidos durante las crisis de 1976 y 1982. Para ello, en el corto plazo, la política económica tendría como objetivo: i) frenar el proceso inflacionario, ii) reducir el déficit de la balanza de pagos y de cuenta corriente, iii) restringir la demanda agregada a través de la reducción del gasto público y, por ende, el aumento de los ingresos fiscales y iv) el deslizamiento del tipo de cambio, véase gráfica 1 y 2.

\section{Gráfica 1}

México: Balanza Comercia

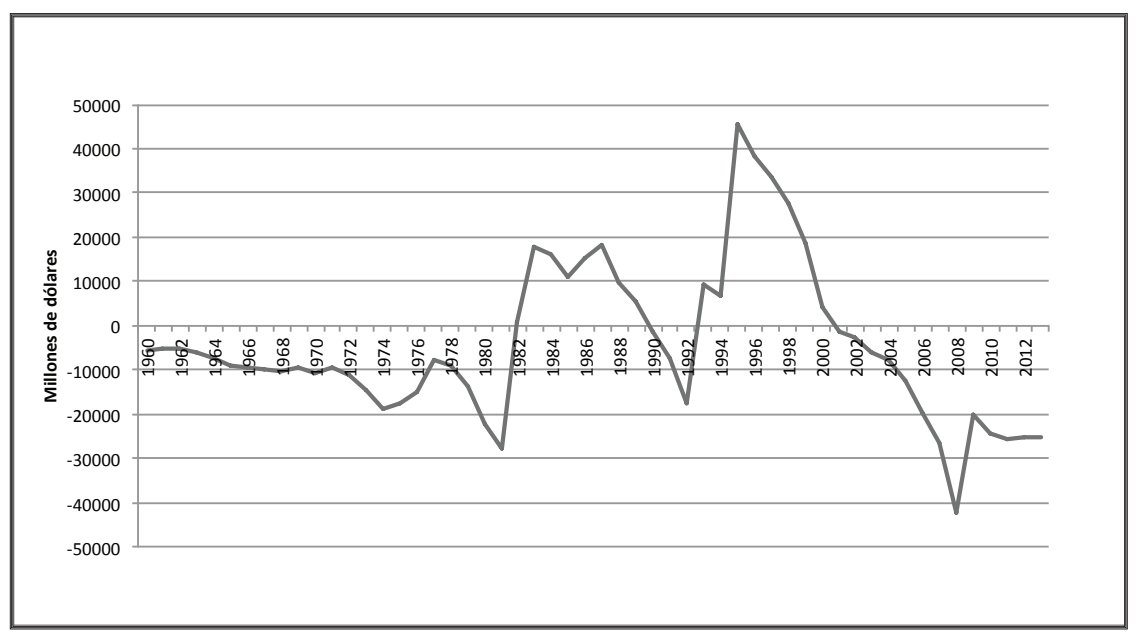

Fuente: elaboración propia con datos de Banco Mundial y OCDE. 
Gráfica 2

México: Superávit/Déficit Presupuestal

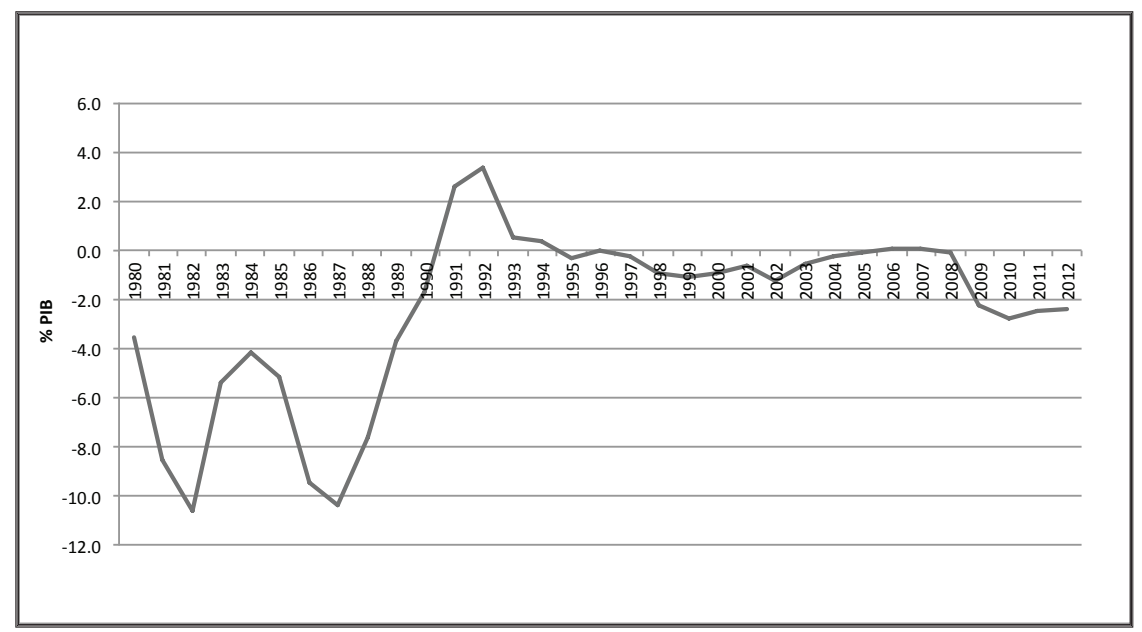

Fuente: elaboración propia con datos de Banco de México.

Mientras que el segundo eje estaría dirigido a la liberalización comercial y la desregulación de la ley de Inversión Extranjera Directa (IED). En este marco, el fundamento de la política económica de mediano y largo plazo sería: a) promover las exportaciones no petroleras, $b$ ) consolidar el sector manufacturero de exportación como núcleo de desarrollo, c) eliminar las barreras arancelarias y no arancelarias al comercio internacional y $d$ ) reducir el déficit de la balanza de pagos y de cuenta corriente (Landa, 2010).

En términos generales el modelo de crecimiento "hacia fuera" ha traído resultados mixtos. En materia macroeconómica los efectos han sido positivos, por un lado, se logró contener el elevado déficit fiscal de los años ochenta, que promediaba 6.4 puntos porcentuales del Producto Interno Bruto (PIB), hasta alcanzar durante la década de los noventa un superávit promedio de $0.3 \%$ del PIB y un moderado déficit presupuestal de $1.1 \%$ del PIB durante el periodo 2000-2013. En cuanto al proceso inflacionario, la transición hacia la estrategia de Política Monetaria de Objetivo de Inflación (PMOI) durante las últimas dos décadas, ha permitido retraer la inestabilidad de la tasa de inflación experimentada durante los ochenta, situando la evolución de este indicador alrededor de un promedio anual de $4.5 \%$. Este entorno de saneamiento fiscal y control inflacionario, ha permitido que la deuda pública descienda de manera 
sostenida (como porcentaje del PIB) desde mediados de la década de los noventa.

Con relación al comercio exterior, si bien el cambio estructural le permitió a la economía alcanzar una exitosa inserción al mercado mundial, basado en el robusto crecimiento y reconversión de las exportaciones, también produjo una fuerte dependencia hacia las importaciones, como consecuencia del patrón de especialización del sistema productivo de exportación (modelo importador-exportador), creándose enormes desequilibrios en la balanza comercial. Desde esta perspectiva, parece que una restricción fundamental del crecimiento económico de largo plazo de la economía mexicana lo constituye la balanza de pagos (ley de Thirlwall).

La expansión de la actividad económica, por otro lado, advierte resultados poco robustos y marginales. Según se observa en el cuadro 1, durante poco más de tres décadas, el ingreso per cápita apenas ha alcanzado un crecimiento promedio anual de $0.3 \%$ y el PIB una tasa del $2.4 \%$ promedio anual. Desempeño enteramente contrastante con la dinámica experimentada durante la vigencia del MIS, donde el PIB y PIB per cápita mantuvieron tasas de crecimiento superiores al $6.5 \%$ y $3.5 \%$, respectivamente.

\section{Cuadro 1}

Comportamiento macroeconómico de la economía mexicana

\begin{tabular}{|c|c|c|c|c|c|c|c|c|}
\hline Periodo & $\begin{array}{c}\text { PIB } \\
\text { per cápita } \\
\text { (Var \%) }\end{array}$ & $\begin{array}{l}\text { PIB } \\
\text { (Var } \\
\% \text { ) }\end{array}$ & $\begin{array}{c}\text { FBKF } \\
(\% \text { PIB) }\end{array}$ & $\begin{array}{c}\text { Exportaciones } \\
\text { (Var \%) }\end{array}$ & $\begin{array}{l}\text { Importaciones } \\
\text { (Var \%) }\end{array}$ & $\begin{array}{c}\text { Tasa de } \\
\text { desempleo } \\
\text { (Var \%) }\end{array}$ & $\begin{array}{c}\text { Inflación } \\
\text { (Var \%) }\end{array}$ & $\begin{array}{c}\text { Tipo de } \\
\text { cambio } \\
\text { nominal } \\
\text { (Var \%) }\end{array}$ \\
\hline $1960-1970$ & 3.5 & 6.8 & 17.6 & 9.4 & 7.5 & - & 2.7 & 0.0 \\
\hline $1970-1980$ & 3.7 & 6.7 & 20.7 & 11.6 & 9.7 & - & 16.5 & 6.3 \\
\hline 1980-1990 & -0.2 & 1.8 & 20.0 & 7.8 & 1.7 & 8.6 & 65.1 & 61.7 \\
\hline $1990-2000$ & 1.7 & 3.6 & 18.4 & 13.5 & 13.0 & -2.2 & 18.3 & 12.9 \\
\hline $2000-2013$ & 0.8 & 2.1 & 21.3 & 3.5 & 4.3 & 6.4 & 4.5 & 2.3 \\
\hline $1960-1980$ & 3.6 & 6.7 & 19.1 & 10.5 & 8.6 & - & 9.4 & 3.1 \\
\hline $1980-2013$ & 0.7 & 2.4 & 20.1 & 7.7 & 6.0 & 4.4 & 24.6 & 21.1 \\
\hline
\end{tabular}

Fuente: elaboración propia con datos de BANXICO, OCDE y Banco Mundial.

\section{Gasto público, inversión y crecimiento en México: evidencia empírica}

El propósito de este apartado es evaluar empíricamente el impacto de la política fiscal, implementada durante los últimos 30 años en la economía mexicana, 
sobre el crecimiento económico. Para este efecto, primero, empleamos el enfoque teórico de Thirlwall (2003), con el fin de demostrar el carácter endógeno del crecimiento $\mathrm{y}$, con ello, el papel de distorsión de la política de disciplina fiscal que crea un entorno de estancamiento estabilizador. Segundo, analizamos la sensibilidad de la inversión privada con relación a la dinámica de la inversión pública, el producto y el tipo de cambio real en el largo plazo. Tercero, examinamos el impacto del gasto del gobierno y del tipo de cambio real sobre los desequilibrios del producto. Cuarto, evaluamos el efecto del gasto del gobierno y la relación capital trabajo sobre la tasa natural de crecimiento.

\subsection{La endogeneidad de la tasa natural: el modelo}

La hipótesis de endogeneidad establece que en el largo plazo el producto potencial, $g_{n}$, se ajusta a las fluctuaciones de la tasa de crecimiento observada $g_{t}$, lo que significa que las variaciones de la demanda agregada determinan simultáneamente la senda de crecimiento observada y la evolución de la tasa natural.

León-Ledesma y Thirlwall (2002) proponen una técnica para estimar la tasa natural y medir la sensibilidad de ésta con respecto a $g_{t}$. En principio, suponen que la tasa de crecimiento observada del producto, $g_{t}$, es una función lineal de la variación porcentual de la tasa de desempleo, $\Delta \% U$ (Thirlwall, 1969), como sigue:

$$
\mathrm{g}_{\mathrm{t}}=\mathrm{a}_{1}-\mathrm{b}_{1}(\Delta \% \mathrm{U})
$$

La tasa natural de crecimiento $g_{n}$, viene definida por el término constante $a_{1}$, cuando $\Delta \% \mathrm{U}=0$; es decir, aquella que mantiene constante la variación porcentual de la tasa desempleo.

Una vez obtenida la tasa potencial, se procede a calcular la desviación entre la tasa de crecimiento observada $g_{t}$, y la tasa natural $g_{n}$. Este proceso conlleva a diferenciar en el modelo la trayectoria durante la fase auge y la fase normal del ciclo económico; mediante la incorporación de una variable dummy, D, a la ecuación, con valor de 1 cuando $g_{t}>g_{n}$ y 0 cuando $g_{t} \leq g_{n}$ :

$$
g_{t}=a_{2}+b_{2}\left(D_{t}\right)-c_{2}(\Delta \% U)_{t}
$$


Efectivamente, la ecuación (2) permite probar formalmente la hipótesis de endogeneidad de la tasa natural, $g_{n}$, durante los periodos de expansión, definida como $g_{n}=a_{2}+b_{2}$. Si la variable ficticia es estadísticamente significativa y $a_{2}+b_{2}>a_{1}$, entonces la tasa de crecimiento observada, $g_{t}$, ejerce un efecto de atracción incorporando más unidades de trabajo al mercado laboral e incrementando la productividad el aumento endógeno al crecimiento del producto y a la acumulación de capital, véase Kaldor, 1957.

Por lo tanto, si el crecimiento de los insumos ${ }^{2}$ y la productividad ${ }^{3}$ son elásticos a las variaciones del producto, las diferencias en las tasas de crecimiento económico entre países puede explicarse con base en las restricciones que imponen las variaciones de la demanda efectiva (León-Ledesma y Thirlwall, 2002). Esta conclusión sugiere que la tasa natural está determinada por la brecha de utilización de la capacidad económica $(G A P){ }_{,}^{4}$ que a su vez depende de la desviación del tipo de cambio real respecto de su nivel de equilibrio, $e_{r}$ y de la tasa de crecimiento del gasto de gobierno, $g g$.

Si consideramos que la tasa natural de crecimiento "es aquella que mantiene constante la tasa de desempleo" (Thirlwall, 2003:109), entonces la tasa natural en periodos normales dependerá en forma positiva del crecimiento de la relación capital por trabajador $(K L)$; así, un aumento (disminución) de esta variable producirá un incremento (reducción) de la tasa de crecimiento que mantendría constante la tasa de desempleo. De esta manera, podemos expresar la endogeneidad de la tasa natural de crecimiento de la siguiente forma:

$$
g_{t}=a_{1}(G A P, K / L)-b_{1}(\Delta \% U)
$$

con :

$$
G A P=f\left(e_{r}, g g\right)
$$

\footnotetext{
${ }^{2}$ Este efecto de la demanda agregada sobre la oferta laboral resulta de los cambios en las decisiones de los trabajadores potenciales, las tasas de participación en la fuerza laboral, las horas-trabajo y los patrones de migración.

${ }^{3}$ La expansión (contracción) de la demanda agregada afecta las decisiones de inversión de las empresas, incidiendo de esta forma en el ritmo de acumulación de capital. Consecuentemente, dependiendo del grado en que el progreso técnico se encuentre incorporado en el capital, la demanda afecta a la tecnología, así como por la acumulación de capacidades de absorción (aprendizaje en la práctica) y la presencia de los rendimientos de escala estáticos y dinámicos.

${ }^{4}$ La brecha de utilización de la capacidad económica se define como la diferencia entre la utilización efectiva u observada de la capacidad productiva instalada y el potencial productivo (producto potencial) de la economía en un periodo dado.
} 
donde $e_{r}$ representa la desviación del tipo de cambio real respecto de su valor de equilibrio; $g g$ la tasa de crecimiento del gasto de gobierno; $K / L$ constituye la relación capital por trabajador; $g g$ la tasa de crecimiento del gasto del gobierno. Se espera que $a_{1 G A P}>0 ; a_{1 K / L}>0 ; f_{e r}>0 ; f_{g g}>0$.

Sustituyendo (4) en (3) y reescribiendo tenemos:

$$
g_{t}=a_{4}\left(e_{r}, g g, K / L\right)-b_{4}(\Delta \% U)_{t}
$$

Donde el término $a_{4}$ es la tasa natural de crecimiento en periodos normales que depende de: $a$ ) la desviación del tipo de cambio real respecto de su valor de equilibrio; $b$ ) de la tasa de crecimiento del gasto de gobierno, y c) de la relación capital por trabajador. Se espera que $a_{4 e r}>0$, $a_{4 g g}>0$ y $a_{4 \mathrm{~K} / \mathrm{L}}>0$.

Con base en lo anterior, es posible afirmar que si la tasa natural de crecimiento en periodos normales depende del GAP y de la relación capital-trabajo $K L$, entonces la elasticidad de la tasa natural de crecimiento económico ${ }^{5}$ en periodos de auge con relación a la de periodos normales, también dependerá del GAP y de $K L$ sólo que en forma negativa:

$$
a_{2}+a_{3}-a_{4}=h(G A P, K / L)
$$

Con base en la ecuación (6) podemos comprobar si la tasa natural de crecimiento en periodos normales está vinculada en forma positiva con la desviación del tipo de cambio real respecto a su valor de equilibrio, ${ }^{6}$ con la tasa de crecimiento del gasto de gobierno y con la relación capital por trabajador; así como si su elasticidad en periodos de expansión con respecto a los periodos normales es una función negativa de la desviación del tipo de cambio real respecto a su valor de equilibrio, de la tasa de crecimiento

\footnotetext{
${ }^{5}$ La elasticidad de la tasa natural de crecimiento se refiere a la variación de ésta en los momentos de expansión cíclica con relación al nivel que la misma tasa natural de crecimiento tiene en los momentos de desempeño normal de la economía. La elasticidad referida, entonces, es la sensibilidad de la tasa natural de crecimiento económico ante las expansiones cíclicas, y depende en forma negativa del GAP y de K/L. ${ }^{6}$ Es decir, si una desviación (por ejemplo, una apreciación) del tipo de cambio con respecto a su valor de equilibrio afecta de manera positiva (expansiva) a la tasa natural de crecimiento en periodos normales.
} 
del gasto de gobierno y de la relación capital por trabajador. Con $h_{G A P}>0$ $h_{K / L}>0$.

\subsection{Demanda agregada, gasto de gobierno, inversión, tasa natural y tipo de} cambio ¿una relación de largo plazo?

En esta sección analizamos primero, si las variaciones de la demanda efectiva y la inversión pública generan un efecto acelerador de la inversión privada. Con este fin, consideramos una especificación donde la inversión privada es función de la brecha del producto y la inversión pública (Vázquez y Avendaño, 2012), de la forma:

$$
\mathrm{ipr}_{t}=\beta_{0}+\beta_{1} g a p_{t}+\beta_{2} i p b_{t}+\varepsilon_{t}
$$

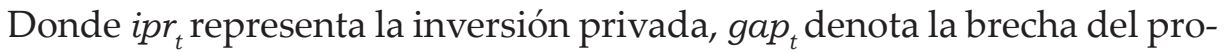
ducto con respecto a su tendencia de largo plazo, $i p b_{t}$ la inversión pública y $\varepsilon_{t}$ el término de error. Se espera que $\beta_{1}>0$ y $\beta_{2}>0$, lo cual indicaría que la inversión pública contribuye a incrementar la rentabilidad de la inversión privada (efecto de atracción versus efecto expulsión).

Asimismo, examinamos el grado de sensibilidad de la brecha del producto a las variaciones del gasto de gobierno y el tipo de cambio real. Para lo cual establecemos la siguiente ecuación:

$$
\operatorname{gap}_{t}=\phi_{0}+\phi_{2} g g_{t}+\phi_{3} q d_{t}+\varepsilon_{t}
$$

Donde $g_{a p}$ constituye la brecha del producto, $g g_{t}$ representa el gasto de gobierno, $q d_{t}$ denota la desviación del tipo de cambio real respecto a su valor de equilibrio y $\varepsilon_{t}$ es el término de error.

Finalmente, consideramos el efecto que la brecha del producto y la relación capital trabajo tiene sobre la tasa natural de crecimiento, durante las fases normales del ciclo y durante las etapas de auge (Vázquez y Avendaño, 2012). En virtud de lo anterior, consideramos las siguientes especificaciones:

$$
g n_{t}=\psi_{0}+\psi_{1} g a p_{t}+\psi_{3} k_{t}+\varepsilon_{t}
$$




$$
g a_{t}=\gamma_{0}+\gamma_{1} g a p_{t}+\gamma_{3} k_{t}+\varepsilon_{t}
$$

Donde $g n_{t}$ y $g a_{t}$ representan la tasa natural de crecimiento en fase normal y de expansión del ciclo económico, respectivamente. Mientras que gap $_{t}$ constituye la brecha del producto y $k_{t}$ la relación capital trabajo. Como antes, las letras minúsculas simbolizan tasas de crecimiento y $\varepsilon_{t}$ el término de error.

\subsection{Resultados econométricos}

En principio, realizamos el análisis de sensibilidad (endogeneidad) de la tasa natural de crecimiento para el caso de México. Con este fin utilizamos el método de regresión recursiva, ${ }^{7}$ con subperiodos de quince años: 1987-2002; 1988-2003; 1989-2004; 1990-2005; 1991-2006; 1992-2007; 19932008; 1994-2009; 1995-2010; 1996-2011; 1997-2012; 1998-2013; 1999-2014. Los datos para la estimación empírica comprenden series trimestrales de enero-marzo de 1987 a julio-septiembre de 2014.

De acuerdo con nuestras estimaciones, la tasa natural de crecimiento, $g_{n}$, aumenta alrededor de 1.7 puntos porcentuales cuando $g_{t}>g_{n}$, equivalente a una elasticidad del $61.4 \%$, lo que implica una transición de la tasa potencial de $2.8 \%$ durante las fases normales del ciclo económico a sendas del $4.5 \%$ durante las fases de expansión económica. Estos resultados sugieren que la tasa natural de crecimiento es endógena a los movimientos de la tasa de crecimiento observada y a las fluctuaciones de la demanda agregada en largo plazo; esto significa que la tasa de crecimiento observada en periodos de auge provoca un crecimiento de la fuerza de trabajo y de la productividad.

Es preciso mencionar que desde finales de la década de los ochenta la tasa natural de crecimiento, tanto en las fases normales del ciclo como en los periodos de expansión, denota un patrón de desaceleración progresiva, tendiente al estancamiento, véase gráfica 3.

\footnotetext{
${ }^{7}$ La técnica de regresión recursiva consiste en estimar una ecuación en varios subperiodos traslapados de igual tamaño.
} 


\section{Gráfica 3}

México: tasa natural de crecimiento y su elasticidad

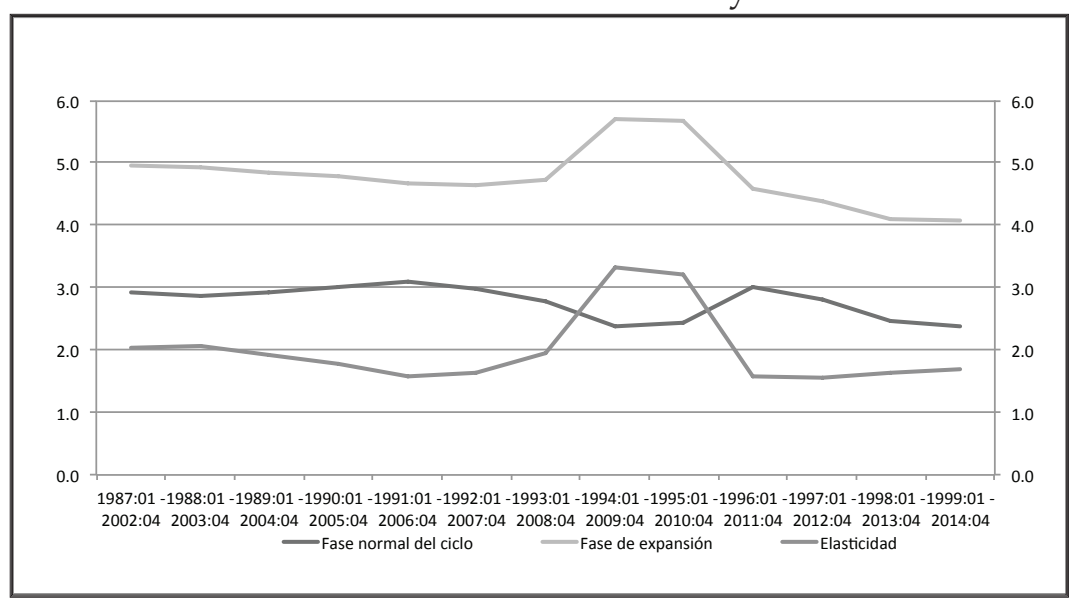

Fuente: cálculos con base en datos de la OCDE.

Para analizar los factores determinantes de la inversión privada empleamos los métodos de Newey-West y mínimos cuadrados generalizados, así como especificación dinámica de la ecuación (7). El objetivo es estimar el impacto de la inversión pública sobre la inversión, a fin de probar la hipótesis de complementariedad entre ambos agregados. La muestra integra series anuales sobre inversión pública, inversión privada y brecha de producto ${ }^{8}$ (desviación del PIB de su tendencia de largo plazo) durante el periodo 19602013.

Las estimaciones indican que la inversión pública genera un efecto positivo sobre la inversión privada, efecto de atracción crowding in. Concretamente, los resultados muestran que un aumento de un punto porcentual en la inversión pública, genera un incremento de la inversión privada de casi un punto porcentual $(0.93 \%)$. Asimismo, la evidencia sugiere un importante efecto acelerador sobre la inversión privada en la medida que aumenta el

${ }^{8}$ Obtenida mediante el filtro de Hodrick-Prescott (HP). Por definición HP separa una serie de tiempo en un componente cíclico $\left(\mathrm{c}_{\mathrm{t}}\right)$ y uno tendencial $\left(\mathrm{g}_{\mathrm{t}}\right)$, esto es $\mathrm{Y}_{\mathrm{t}}=\mathrm{g}_{\mathrm{t}}+\mathrm{c}_{\mathrm{t}}$. El proceso de optimización seguido por HP establece:

$$
\min _{\left\{g_{t}\right\}_{t=-1}^{T}}\left\{\sum_{t=1}^{T} C_{t}^{2}+\lambda \sum_{t=1}^{T}\left[\left(g_{t}-g_{t-1}\right)-\left(g_{t-1}-g_{t-2}\right)\right]^{2}\right\}
$$

Donde $\lambda$ corresponde a un factor de suavización. Basados en la evidencia y pertinencia de los factores de suavizado, los autores sugieren para datos anuales $\lambda=100$ y $\lambda=1,600$ para datos trimestrales. 
PIB observado. Según nuestras estimaciones, un aumento del 1\% en la brecha del producto produce un incremento de 3.6\% en la inversión privada, véase cuadro 2.

En general, las pruebas de significancia conjunta e individual sugieren que las variables de cada sistema son estadísticamente diferentes de cero, con un nivel de confianza del 95\%; y los modelos producen términos de error I (0).

Cuadro 2

Determinantes de la inversión privada

\begin{tabular}{|c|c|c|c|}
\hline \multirow{2}{*}{ Coeficiente } & Modelo (8) & Modelo (7) & Modelo $(7)^{c}$ \\
\hline & $i p r_{t}$ & $i p r_{t}$ & $i p r_{t}$ \\
\hline \multicolumn{4}{|l|}{ Largo plazo } \\
\hline Constante & $\begin{array}{c}1.3906 \\
{[0.049]^{*}}\end{array}$ & $\begin{array}{c}2.8835 \\
{[0.034]^{*}}\end{array}$ & $\begin{array}{l}11.2126 \\
{[0.006]^{*}}\end{array}$ \\
\hline $\operatorname{gap}_{t}$ & $\begin{array}{c}3.6055 \\
{[0.000]^{*}}\end{array}$ & $\begin{array}{c}1.8516 \\
{[0.002]^{\star}}\end{array}$ & $\begin{array}{r}-10.7449 \\
{[0.145]} \\
\end{array}$ \\
\hline$i p b_{t}$ & $\begin{array}{c}0.9341 \\
{[0.000]^{*}}\end{array}$ & $\begin{array}{c}0.9338 \\
{[0.000]^{*}}\end{array}$ & $\begin{array}{c}0.6939 \\
{[0.000]^{*}}\end{array}$ \\
\hline$q_{t}$ & - & $\begin{array}{c}-0.3103 \\
{[0.013]^{*}}\end{array}$ & $\begin{array}{l}-1.3797 \\
{[0.006]^{*}}\end{array}$ \\
\hline \multicolumn{4}{|l|}{ Corto plazo } \\
\hline$i p r_{t-1}$ & - & - & $\begin{array}{l}-0.1642 \\
{[0.023]^{\star}}\end{array}$ \\
\hline$\Delta i p b_{t}$ & - & - & $\begin{array}{c}0.5362 \\
{[0.066]^{\star *}}\end{array}$ \\
\hline$\Delta \operatorname{gap}_{t}$ & - & - & $\begin{array}{c}3.3309 \\
{[0.000]^{\star}}\end{array}$ \\
\hline$\Delta \operatorname{gap}_{t-1}$ & - & - & $\begin{array}{c}1.5342 \\
{[0.005]^{\star}}\end{array}$ \\
\hline Prueba F & $\begin{array}{l}204.40 \\
{[0.0000]^{*}}\end{array}$ & $\begin{array}{l}910.97 \\
{[0.0000]^{*}}\end{array}$ & - \\
\hline$R^{2}$ & - & 0.5690 & 0.6930 \\
\hline
\end{tabular}

a/ Regresión basada en el método Newey-West.

b/ Regresión basada en mínimos cuadrados generalizados, estimador de Prais-Winsten (método de Cochrane-Orcutt).

c/ Modelo autorregresivo con rezagos distribuidos, ARDL (p, $q, \mathrm{q})$.

*Significativo al 5\%**Significativo al 10\%. Valor-p entre corchetes.

Fuente: estimaciones con base en información Banco Mundial, INEGI, OCDE y Banco de México. 
Los resultados no varían significativamente cuando se incluye el tipo de cambio real a la ecuación (7). Como antes, se encuentra un efecto de complementariedad entre inversión pública y privada, así como una nítida sensibilidad de la inversión ante el aumento del ingreso.

En cuanto a la especificación dinámica de la función de inversión privada, basada en un modelo de sistema autorregresivo con rezagos distribuidos (ARDL), el sentido de los signos de la inversión pública y del tipo de cambio real no cambia. Debemos anotar que la inclusión de pocos rezagos en el modelo, indica que el comportamiento dinámico proviene del término de corrección de error. En este sentido, el coeficiente de ajuste posee el signo correcto, lo cual confirma la presencia de un co-movimiento de largo plazo entre las variables del sistema.

Nuestros resultados son consistentes con la evidencia hallada en otros estudios. Castillo y Herrera (2005) realizan una estimación econométrica de la relación entre la inversión pública y la inversión privada; encuentran que si bien la inversión pública desplaza a la inversión privada en el corto plazo, efecto de esparcimiento, en el largo plazo la complementa. Esto se debe a que los inversionistas privados aprovechan las externalidades positivas que genera la inversión pública, debido a que ésta se dirige hacia el desarrollo de proyectos de infraestructura. Estos proyectos, una vez terminados, empiezan a diseminar todos sus beneficios, spillover effect, de manera tal que impactan e incentivan los proyectos privados de inversión. Es por ello que, si existe un efecto desplazamiento significativo, éste sería transitorio o de corto plazo, durará el mismo tiempo en que se está financiando la inversión pública. Es decir, que en el largo plazo la inversión pública complementa a la inversión privada.

Con datos trimestrales para el periodo 1980-2007, Fonseca (2009) examina la relación de largo plazo entre la inversión privada, el PIB, la inversión pública, la tasa de interés y la deuda pública interna para el caso de la economía mexicana. Basado en la metodología de vectores autorregresivos con corrección de error (VEC) y sistemas (ARDL), encuentra un efecto de desplazamiento parcial en el corto plazo, mientras que en el largo plazo el efecto es de complementariedad marginal. Estos impactos diferenciados, concluye, dependen esencialmente de la composición de la inversión pública, ya que en voz del autor no es lo mismo invertir en infraestructura y telecomunicaciones que en edificios de uso administrativo.

En otro estudio para México, Gutiérrez (2012) analiza el impacto del gasto primario y del PIB sobre la inversión privada, durante el periodo 1980-2007. 
Sus estimaciones, basadas en un modelo ARDL(4), sugieren que el gasto primario y el PIB producen un efecto positivo sobre los movimientos de la inversión privada, lo que exhibe el papel de atracción de la inversión pública.

Los resultados de la ecuación (8), por otra parte, sugieren que en el largo plazo por cada punto porcentual de depreciación del tipo de cambio real la brecha del producto decrece aproximadamente $0.05 \%$. Mientras que un aumento en $1 \%$ del gasto del gobierno producirá un aumento de $0.08 \%$ en el PIB (ampliación de la brecha del producto), como se puede observar en el cuadro 3.

Cuadro 3

Determinantes de la brecha del producto

\begin{tabular}{|c|c|c|c|}
\hline Coeficiente & $\begin{array}{c}\text { Modelo (8) } \\
\operatorname{gap}_{t}\end{array}$ & $\begin{array}{c}\text { Modelo (9) } \\
g n_{t}{ }^{\prime d} \\
\end{array}$ & $\begin{array}{c}\text { Modelo (10) } \\
g a_{t} / d\end{array}$ \\
\hline Constante & $\begin{array}{c}5.0973 \\
{[0.001]^{*}}\end{array}$ & $\begin{array}{l}-0.4571 \\
{[0.063]^{* *}}\end{array}$ & $\begin{array}{c}0.0416 \\
{[0.860]}\end{array}$ \\
\hline$g g_{t}$ & $\begin{array}{c}0.0846 \\
{[0.070]^{* *}}\end{array}$ & - & - \\
\hline$q d_{t}$ & $\begin{array}{l}-0.04979 \\
{[0.000]^{*}}\end{array}$ & - & - \\
\hline $\operatorname{gap}_{t}$ & - & $\begin{array}{c}0.4329 \\
{[0.074]^{* *}}\end{array}$ & $\begin{array}{c}0.6961 \\
{[0.005]^{*}}\end{array}$ \\
\hline$k_{t}$ & - & $\begin{array}{c}0.0430 \\
{[0.048]^{*}}\end{array}$ & $\begin{array}{r}0.0001 \\
{[0.995]}\end{array}$ \\
\hline rho & - & - & 0.4314 \\
\hline Prueba F & $\begin{array}{l}9.58 \\
{[0.0003]^{*}}\end{array}$ & $\begin{array}{l}3.93 \\
{[0.0340]}\end{array}$ & $\begin{array}{c}4.85 \\
{[0.0180]}\end{array}$ \\
\hline$R^{2}$ & 0.2770 & - & - \\
\hline
\end{tabular}

a/ Regresión basada en el método Newey-West.

b/Estimador Prais-Winsten (método de Cochrane-Orcutt). Mínimos Cuadrados Generalizados.

c/ Modelo Autorregresivo con Rezagos Distribuidos.

d/ Se estima mediante el método "rolling regression".

*Significativo al 5\% **Significativo al 10\%. Valor-p entre corchetes.

Fuente: estimaciones con base en información Banco Mundial, INEGI, OCDE y Banco de México.

Finalmente, según nuestras estimaciones, encontramos que un aumento del ingreso, así como en la relación capital-trabajo, produce un efecto acelerador 
en la dinámica de la tasa natural de crecimiento. Esto sugiere que una desaceleración del ingreso (GAP) y de la inversión pública contrae la inversión privada, afectando de esta forma la tasa de crecimiento anual y, por ende, la tasa natural de crecimiento.

\section{Conclusiones}

Los resultados obtenidos mediante el análisis empírico muestran que en la economía mexicana la política fiscal practicada en la forma de disciplina de finanzas públicas "sanas" ha tenido un impacto negativo en la inversión productiva, en el gasto del gobierno y, por consecuencia, en la tasa de crecimiento económico. Además, la evidencia empírica muestra que la inversión es sensible y elástica ante choques del gasto del gobierno.

Con este análisis se demostró la importancia de la política fiscal en la determinación de la tasa de crecimiento de la economía de nuestro país. Por un lado, la tasa natural de crecimiento en periodos normales está determinada por el GAP y por la tasa de crecimiento de la razón $K / L$; por lo cual la disminución del gasto de gobierno y la apreciación del tipo de cambio real respecto a su nivel de equilibrio, ambos determinantes positivos del GAP, deprimen la tasa natural de crecimiento en periodos normales. Por otro lado, la disminución del GAP y de la inversión pública contrae la inversión privada, lo cual afecta la tasa de crecimiento anual de la razón $K / L$, originándose así otra fuente de contracción de la tasa natural de crecimiento en periodos normales.

Los resultados de las pruebas realizadas permiten concluir que el producto potencial de la economía mexicana ha disminuido debido al deterioro de la inversión pública y también de la inversión privada. Esto se explica por el deterioro de la capacidad económica, debido al mismo tiempo a la reducción del gasto del gobierno y a la apreciación del tipo de cambio real.

La tasa de crecimiento del producto potencial se ha deteriorado también por la disminución del stock de capital, el cual responde a las variaciones de la inversión. Igualmente se observa un régimen de baja inversión en la economía, en parte a causa de la caída significativa del gasto del gobierno, en particular a la disminución de la inversión pública. Como se ha comentado, otros estudios empíricos han encontrado que la inversión pública tiene un efecto complementario sobre la inversión privada en el largo plazo (Castillo y Herrera 2005), lo cual es consistente con el significado de nuestros 
resultados. Adicionalmente, la caída de la inversión privada responde a la disminución del GAP, puesto que existe una relación directa entre el GAP y la tasa de crecimiento de la inversión privada.

Asimismo, tanto el GAP como la inversión pública contribuyen al crecimiento de la inversión privada, mientras que el gasto público y las desviaciones del tipo de cambio respecto de su nivel de equilibrio han influido en el comportamiento del GAP de la economía mexicana. Además, dado que la tasa de crecimiento natural de la economía mexicana es endógena con respecto a la demanda agregada, parece claro que la política de finanzas públicas "disciplinadas" en México han jugado un papel significativo en la evolución reciente de la economía, cuyo desempeño ha seguido un patrón que hemos caracterizado como de estancamiento estabilizador. Por lo tanto, parece razonable proponer que la sustitución del enfoque actual de balance fiscal anual -que impone una restricción presupuestaria con efectos depresivos sobre la actividad económica- en favor de un modelo alternativo de política fiscal, podría contribuir a estimular el empleo y el crecimiento económico con estabilidad de precios.

Finalmente, la evidencia empírica que hemos evaluado en este trabajo revela que, en efecto, la tasa de crecimiento natural de la economía mexicana es endógena con relación a la demanda agregada, que es lo que pretendíamos comprobar. Por tanto, dado este resultado fundamental, es razonable proponer que el abandono del enfoque ortodoxo de finanzas "sanas" y la adopción de un régimen de política fiscal heterodoxo puede contribuir tanto a la disposición de mecanismos contracíclicos, cuanto a estimular y optimizar el crecimiento económico con estabilidad de precios y pleno empleo. La salida de la trampa de desarrollo en que se encuentra estancada la economía mexicana requiere de un modelo alternativo de política fiscal. 


\section{Referencias}

Ahmed, H. y S. Miller (2000). "Crowding-out and crowding-in effects of the components of government expenditure", Contemporary Economic Policy, 18 (1), pp. 124-33.

Arestis, P. y M. Sawyer (2003). Reinventing Fiscal Policy, The Levy Economics Institute of Bard College, Working Paper No. 381. (2003). On the Effectiveness of Monetary Policy and Fiscal policy, The Levy Economics Institute of Bard College, Working Paper, No. 369.

(2003b). The Case for Fiscal Policy, The Levy Economics Institute of Bard College, Nueva York, Working Paper, No. 382.

(2003c). Reinventing Fiscal Policy, The Levy Economics Intitute of Bard College, Nueva York, Working, No. 381.

Aspe, Pedro (1993). Economic Trnasformacion, The Mexican Way, The MIT Press.

Blinder, A. S. (2006). "The case against the case against discretionary fiscal policy", Kopcke R. W.; G. M. B. Tootell y R. K. Triest, eds., The Macroeconomics of Fiscal Policy, Cambridge MA, The MIT Press.

Castillo Ponce, R. y J., Herrera Hernández (2005). “Efecto del gasto público sobre el gasto privado en México", Estudios Económicos, vol. 20, núm. 2, El Colegio de México, A.C., pp. 173-196.

Dray, M. y A:P: Thirll (201). "The Endogeneity of the Natural Rate of Growth for a Selection of Asian Countries", Journal of Post-Kernesian Economics, en prensa.

Fonseca, F. (2009). "El impacto de la inversion pública sobre la inversion privada en México, 1980-2007", Estudios Económicos, vol. 24, núm. 2 (Julio-diciembre), El Colegio de México, A.C., pp. 127-284.

Gutiérrez, C. F. S. (2012). El efecto de la inversion pública sobre la inversion privada: el caso de México 1980-2007. tesis de maestría. FLACSO, México.

Harrod, Roy (1939). “An Essay in Dynamic Theory", Economic Journal, 49 (193), pp. 14-33.

Ibarra, Carlos (2008). La paradoja del crecimiento lento de México, Revista de la CEPAL núm. 95, México, pp. 83-103.

Kaldor, Nicholas (1957). "A model of economic growth", Economic Journal, 67 (268), pp. 591-624.

Keynes, J:M: (1936). The General Theory of Employment, Moneyand Interest, 7a. ed., Nueva York, Haycourt \& jovanovich. 
Lanzafame, M. (2010). “The Endogeneity of the Natural Rate of Growth in the Regions of Italy", International Review of Applied Economics, 24 (5), pp. 533-552.

Landa, Heri O. (2010). Crecimiento económico y apertura económica en México: el rol de las externalidades y las capacidades tecnológicas, tesis doctoral. Universidad Autónoma Metropolitana.

León-Ledesma , M. y M. Lanzafame (2010) “The endogenous nature of the 'natural' rate of growth", Setterfield, M. editor, Handbook of Alternative Theories of Economic Growth, Cheltenham, R.U., Edward Elgar, pp. 208-219.

León-Ledesma, M. y A. P. Thirlwall (2000) "Is the natural rate of growth exogenous?", Banca Nazionale del Lavoro Quarterly Review, 53, pp. 433-446.

_ (2002). "The Endogeneity of the Natural Rate of Growth", Cambridge Journal of Economics, 26(4) pp. 441-459.

Libanio, G. (2009) "Aggregate Demand and the Endogeneity of the Natural Rate of Growth: Evidence from Latin American Economies", Cambridge Journal of Economics, 33(5) pp. 967-984.

Lustig, Nora (1992). México the Remaking of an Economy, The Brookings Insitution. Mayer, Jurg (2008). "Policy Space: What, for what, and where?", UNCTAD Discussion Papers No. 191, octubre.

Moreno-Brid, Juan Carlos y Jaime Ros (2009). Development and Growth in the Mexican economy, a historical perspective, Nueva York, Oxford University Press.

OCDE, Tax Revenue Trends 1965-2007.

Perrotini, I. y D. Tlatelpa (2003) Crecimiento Endógeno y Demanda en las Economías de América del Norte, Momento Económico, núm. 128, pp. 10-15.

Phillips, A. W. H. (1958). "The Relationship between Unemployment and the Rate of Change of Money Wages in the United Kingdom 1861-1957", Economica, vol. 25, issue 100, november, pp. 283-299.

Rodrik, D., 2004, "Rethinking growth strategies", WIDER Annual Lecture 8, United Nations World Institute for Development Economic Research, Helsinki.

Sachs, J. y R. Larrain (1994). Macroeconomía en la economía global, México: PrenticeHall, p. 196.

Samuelson, P. A. (1947). Foundations of Economic Analysis, Cambridge, MA., Harvard University Press.

Shaikn, A. y J. Moudud (2004). "Measuring Capacity in OECD Countries: A Cointegration Method", Working Paper No. 415, The Levy Economics Institute, November.

Solow (1956). "A Contribution of the Theory of Economic Growth", Quarterly Journal of Economics, vol. 70, pp. 65-94. 
Thirlwall, A. P. (1969). "Okun's Law and the natural rate of growth", The Southern Economic Journal, 36, pp. 87-89. 1994, Growth and Development, Londres, MacMillan.

Thirlwall, A. P. 2003, La naturaleza del crecimiento económico. Un marco alternativo para comprender el desempeño de las naciones, FCE, México.

Vázquez M., J. A. y B. L. Avendaño V., (2012). “¿Modelo de crecimiento exportador o modelo de estancamiento exportador? El caso de México, 1961-2010", Investigación Económica, vol. LXXI núm. 282, octubre-diciembre, pp. 93-108.

Vogel, L. (2009) 'The Endogeneity of the Natural Rate of Growth: An Empirical Study for Latin American Countries', International Review of Applied Economics, 23(1) pp. 41-53.

Williamson, J. (2003). “Overview: En agenda for restarting growth and reform", After the Washington Consensus, Kuczynski y Wiliamson. Ed. Washintogton, D. C. Intitute for International Economics.

Páginas electrónicas: www.banxico.org.mx; www.inegi.org.mx; www.shcp.gob. mx; www.cepal.org; www.worldbank.org. 